\title{
BMJ Open Companion robots for older people: importance of user-centred design demonstrated through observations and focus groups comparing preferences of older people and roboticists in South West England
}

Hannah Louise Bradwell, ${ }^{\oplus 1}$ Katie Jane Edwards, ${ }^{\circledR}$ Rhona Winnington, ${ }^{\circledR}$ Serge Thill, ${ }^{\oplus 3}$ Ray B Jones ${ }^{\oplus 1}$

To cite: Bradwell HL, Edwards KJ, Winnington R, et al. Companion robots for older people: importance of usercentred design demonstrated through observations and focus groups comparing preferences of older people and roboticists in South West England. BMJ Open 2019;9:e032468. doi:10.1136/ bmjopen-2019-032468

- Prepublication history and additional material for this paper are available online. To view these files, please visit the journal online (http://dx.doi org/10.1136/bmjopen-2019032468).

Received 25 June 2019 Revised 05 September 2019 Accepted 09 September 2019

D) Check for updates

(C) Author(s) (or their employer(s)) 2019. Re-use permitted under CC BY-NC. No commercial re-use. See rights and permissions. Published by BMJ.

For numbered affiliations see end of article.

\section{Correspondence to} Hannah Louise Bradwell; hannah.bradwell@plymouth. ac.uk

\section{ABSTRACT}

Objective Companion robots, such as Paro, may reduce agitation and depression for older people with dementia. However, contradictory research outcomes suggest robot design is not always optimal. While many researchers suggest user-centred design is important, there is little evidence on the difference this might make. Here, we aimed to assess its importance by comparing companion robot design perceptions between older people (end users) and roboticists (developers).

Design Older people and roboticists interacted with eight companion robots or alternatives at two separate events in groups of two to four people. Interactions were recorded, participants' comments and observations were transcribed, and content was analysed. Subsequently, each group participated in focus groups on perceptions of companion robot design. Discussions were recorded and transcribed, and content was analysed.

Participants and settings Seventeen older people (5 male, 12 female, ages 60-99) at a supported living retirement complex, and 18 roboticists (10 male, 8 female, ages 24-37) at a research centre away-day.

Results We found significant differences in design preferences between older people and roboticists. Older people desired soft, furry, interactive animals that were familiar and realistic, while unfamiliar forms were perceived as infantilising. By contrast, most roboticists eschewed familiar and realistic designs, thinking unfamiliar forms better suited older people. Older people also expressed desire for features not seen as important by developers. A large difference was seen in attitude towards ability to talk: $12 / 17(71 \%)$ older people but only 2/18 (11\%) roboticists requested speech. Older people responded positively towards life-simulation features, eye contact, robot personalisation and obeying commands, features undervalued by roboticists. These differences were reflected in preferred device, with 'Joy for All' cat chosen most often by older people, while roboticists most often chose Paro.

Conclusion The observed misalignment of opinion between end users and developers on desirable design
Strengths and limitations of this study

- Novel direct comparison between older people (end users) and roboticists (developers).

- The participation of older people themselves contrasts with previous research using care provider opinions as proxy.

- The range of robots and toys, some specifically designed for older people, extends to previous studies with a limited array of robot features.

- The short interaction time of 10 minutes between participants and robots allowed limited time for familiarity with devices.

- Small sample size (although in-depth qualitative analysis does allow for increased confidence in results, and smaller group size may have limited the influence of social desirability bias or group dynamics).

features of companion robots demonstrates the need for user-centred design during development.

\section{BACKGROUND}

Life expectancy, and thus proportion of the population at retirement age or above, is increasing worldwide. ${ }^{1}$ As human functional capacity can decline with age, ${ }^{2}$ this creates a greater demand for services ${ }^{3}$ while the number of health and social care workers decreases, ${ }^{1}$ putting pressure on health and social care resources. ${ }^{4}$ Steptoe et a $a \tilde{~}$ suggested a growing need for research on maintaining well-being: while supporting physical functioning is often addressed, the psychological health of the ageing population has received less attention. ${ }^{6}$ Assistive robotics, whether rehabilitation or social robots, ${ }^{7}$ could help 
in this respect and alleviate some pressure on health and social care resources. ${ }^{3}$

Here, we consider companion robots-a subset of social robots often designed congruent with animal aesthetics and behaviours ${ }^{78}$ - that alleviate issues of traditional animal-assisted therapy ${ }^{9}$ including reducing risks for the animals themselves. ${ }^{910}$ A prominent example is Paro, the robot seal. ${ }^{10}$ Research has suggested numerous benefits of interacting with Paro, including reduced agitation and depression in dementia, ${ }^{11}{ }^{12}$ more adaptive stress response, ${ }^{13}$ reduced care provider burden, ${ }^{13}$ and significantly improved affect and communication between patients with dementia and day care staff. ${ }^{14}$ Paro may additionally reduce psychoactive and analgesic medication use ${ }^{15}$ and even decrease blood pressure. ${ }^{16}$

These positive results, however, have been questioned. ${ }^{17}$ A comparison between an active Paro and an inactive one found benefits of the active robot were limited to engagement. ${ }^{18}$ One study ${ }^{19}$ found no significant improvement for depression (seeing a significant decrease only for loneliness); another ${ }^{20}$ compared live dog visits to Paro sessions over 6 weeks and found no improvement for depression with either intervention. Research assessing suitability of Paro for a dementia unit suggested it required adaptions; for example, its vocalisations can be distressing. ${ }^{21}$ Finally, a large randomised controlled trial (RCT) found considerable variation in responses to Paro. ${ }^{22}$

While this disparity may result from individual variability, it is also possible robot design factors may be impairing wider acceptance. Similar differences have been observed for other devices; for example, research on AIBO has both shown good acceptability ${ }^{23}$ and found that it encouraged less interaction than a soft toy. ${ }^{24}$ Meanwhile, a review of acceptability towards robots used in aged care suggests a number of robots have failed. ${ }^{3}$

The Almere model of acceptability of social robots among older people strongly suggests acceptability can impact intention to use and therefore actual use of a device. ${ }^{25}$ Furthermore, using robots in contexts they were not designed for can perpetrate negative perceptions of them and reduce acceptability, ${ }^{4}$ which may explain some of the conflicting results on robot companions. User-centred design, in general, thus requires designers to have a deep understanding of those they design for and to involve them in all stages of the process. ${ }^{26}$

Considering perceived requirement can vary between stakeholder groups, ${ }^{27}$ as can technology acceptance, ${ }^{28}$ design requirements likely differ between varied groups of end users, for example, those with physical impairments, ${ }^{29}$ children ${ }^{30}$ or older people. Research should thus be specific to the aim of each robotic system. Generally, integrating user requirements and experiences into design can be difficult. ${ }^{29}$ One challenge noted by Chammas et $a l^{26}$ is the acceptance, recognition and incorporation of user-centred design in practice. Therefore, considering the potential additional effort required, evidence establishing the value of this approach might help encourage designers to adopt this type of methodology.
While little appears to be currently known about how older people perceive robots, ${ }^{31}$ one study explored the meaning behind robotic pets with 41 independent older people ${ }^{32}$ finding that robotic pets could provide social entertainment and interactions. While functional support was appealing, the fiction of robotic comfort was a potential tension. ${ }^{32}$ Participants reported preference for soft fur and suggested play features as an improvement, currently absent from available companion robots. A limitation was the use of unfamiliar, often brightly coloured, child-orientated pets, restricting the range of features participants could inform perceptions on.

More generally, while older people and people with dementia are implicated in companion robot design, they are often not involved, ${ }^{33}$ even given a clearly identified need for ensuring devices adequately meet the needs of the end users. ${ }^{4}$ Instead, older people are often assigned stereotypical needs. ${ }^{33}$ When they are involved, it is usually through care providers and at the end of the design process. $^{32}$

Here, we therefore investigate any notable differences in opinion between 'robot users' and 'robot creators' regarding the design of companion robots and provide initial insights into older peoples' design requirements. The different perceptions between designers and end users we document also demonstrate the importance of user-centred design.

\section{METHODS \\ Design}

This study was one of many substudies forming a doctoral collaborative action research (CAR) project. We conducted observations of roboticists and older people separately interacting with a variety of robots, providing a comprehensive range of features for comparison. Both groups then participated in focus group discussions informed by their interaction experience.

\section{Patient and public involvement}

Due to the wider projects' CAR approach, key stakeholders have been continually involved in designing studies forming this doctoral project. Stakeholders have included older people, family members and health and social care professionals, including dementia liaison services, psychologists and care home management and staff. The older people involved in this study subsequently provided feedback on methods for future research.

\section{Participants and settings}

In total, 35 participants collaborated: 17 older people ( 5 male, 12 female, age range 60-99 years) and 18 roboticists (10 male, 8 female, age range $24-37$ ). Older people were recruited at a supported living complex that houses individuals of and above retirement age within apartments, with a manager present on site. Roboticists were recruited at an away-day event of researchers from a robotics research centre. These included research students, 
academics and individuals developing and researching robotics and social robots, many within the health and social care field. The researchers were therefore familiar with this field, and the students may represent a next generation of developers.

\section{Procedure}

In both settings, participants gave written informed consent, then formed groups of up to four people. Each group moved through three interaction stations where participants engaged in free interaction with a selection of robots or toys. Each station provided a different range of robot/toy features, aesthetics and abilities (figure 1), and was filmed using two cameras. Non-interactive toys and devices with varying sophistication were included in comparison with the high sophistication levels of robots such as Paro. Participants spent $10 \mathrm{~min}$ at each station, with researchers present to assist and answer questions.

After free interaction with all available robots and toys, the participants engaged in semistructured focus group discussions, guided by key questions (box 1). Questions were informed by previous research, ${ }^{34}$ amended only to include more features of interest and to ensure relevance with end users as opposed to care providers. Finally, the participants were debriefed.

Robots starting positions at each station (eg, see figure 2) were randomised, from left to right, to avoid introduction of bias. Researchers maintained a conscious effort to keep interaction unbiased, refraining from leading questions and restricting their role to introducing animals and answering questions during free interactions. The procedure was maintained as much as possible between both settings. Roboticists were asked to think of the target audience of older people when responding to the key questions.

\section{Materials}

In addition to video recordings, field notes, paper participant information sheets, consent forms and debriefs were collected.

\section{Data analysis}

Discussions at all stations were transcribed verbatim and were analysed by two researchers (HB and KE). There were two sets of data for each setting: (1) unprompted opinions based on comments and discussions during free interaction with the range of robots and toys and (2) focus group responses. Both sets of data were analysed separately with NVivo using content analysis to garner emerging themes. Content analysis was selected for inclusion of frequencies of theme occurrence ${ }^{35}$ and involved systematic coding and categorising of text to garner trends, frequencies and relationships of words in discourse. ${ }^{36}$ Researchers undertook a process of data immersion, coding, grouping codes, generating categories and reporting, as prescribed by Elo and Kyngäs. ${ }^{37}$

The results are reported in three sections:

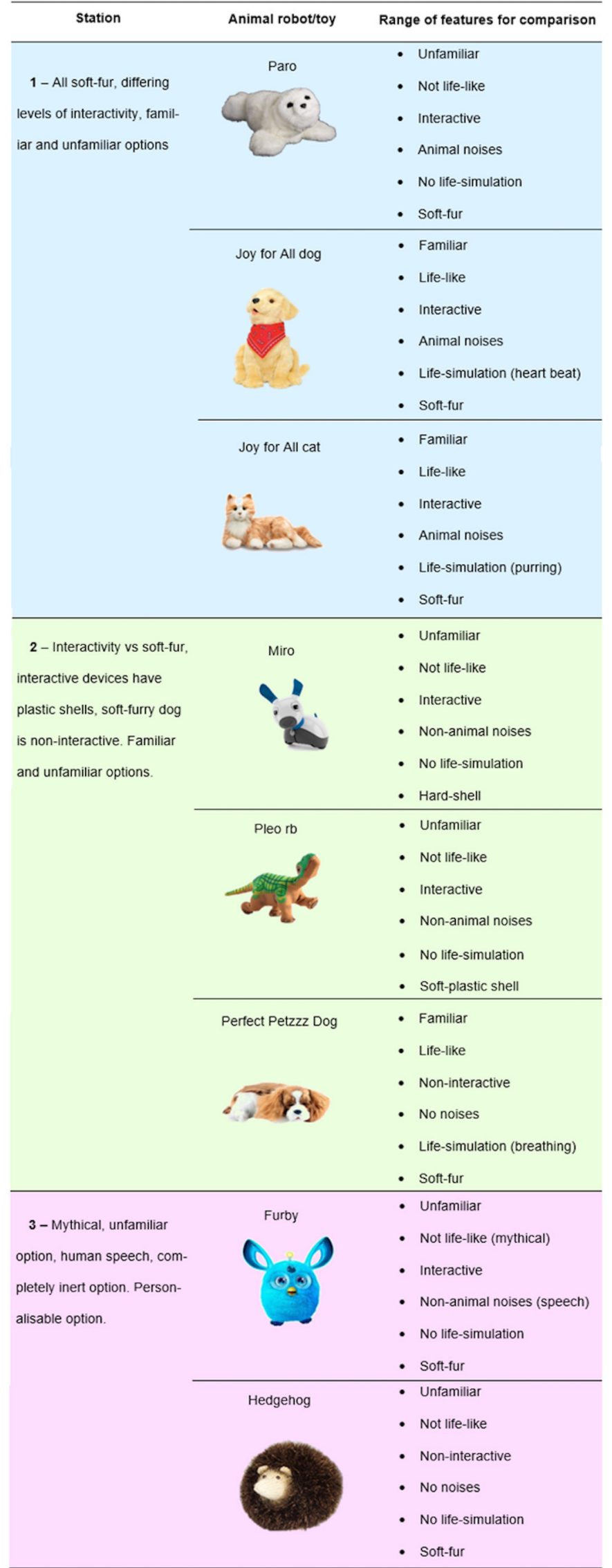

Figure 1 Robots and toys at each interaction station and the associated features for comparison. 


\section{Box 1 Key questions used to guide focus group}

discussions

\section{Key questions}

1. Which of the animals did you like? What is it about those animals that makes you like them?

2. Thinking of designing a new robot for older people, what possibilities and properties should a suitable pet robot have (eg, look, feel and abilities)?

a. What features and qualities are necessary?

b. What features and qualities are desirable?

c. Which expressions are important?

d. Why?

3. What possibilities and properties should a suitable pet robot not have?

4. How do you feel about a companion robot speaking and having a basic conversation?

5. The hedgehog is handmade. What are your thoughts on personalising robots and individuals designing or creating for personal preference of looks, feel and type of animal?

6. What do you think about how realistic or unrealistic the animal should be? How would you feel about a mythical animal?

7. How do you feel about life-simulation features?

8. Would you fancy having one of these animals yourself to keep? Which one would you choose? (For roboticists, which one would you choose for an older person?)

- Section 1 provides the themes arising during content analysis of older people's free interactions, giving initial insight into end-user requirements.

- Section 2 focuses on the themes from focus group discussions and features most commonly discussed by both groups in response to the key questions (box 1).

- Section 3 maps the relationship between older people's unprompted opinions and their focus group responses.
RESULTS

\section{Section 1: content analysis of older people's free interaction} with the robots

This section provides an in-depth exploration of themes, both positive and negative, arising during unprompted, free interactions between older people and the comprehensive range of companion robots. These themes were interactivity, familiarity, shell design and ownership.

\section{Interactivity}

The interactivity theme emerged on 185 occasions through codes: interactivity, speech and talking, commanding the robot, fun, noises and interactivity lacking, strongly suggesting that during live, unprompted interactions, older people demonstrated preference for interactive devices over non-interactive alternatives. The results also indicated eye contact and obeying commands and speech could be improvements on currently available devices.

Interactivity elicited positive comments from participants, such as 'fascinating' (older person (OP) 15), and provided a sense of achievement when a device appeared responsive, such as 'I got the cat to roll over!' (OP16). he participants demonstrated most enjoyment when robots appeared reactive to the individual themselves, rather than producing random movements or sounds, such as 'fun isn't it!' (OP6). In contrast, non-interactive devices provoked negative responses. The Perfect Petzzz dog was described as 'a bit of a disappointment' (OP6), as the dog 'doesn't do much' (OP16), which may become 'boring' (OP12) as 'you can't do more than pat its head' (OP17). Perhaps surprisingly, the participants also underappreciated the interactivity of Paro. The Joy for All animals were seen as highly interactive, despite having more limited technological features, while Paro was described as 'on strike' (OP7) because the participants felt it 'just moves

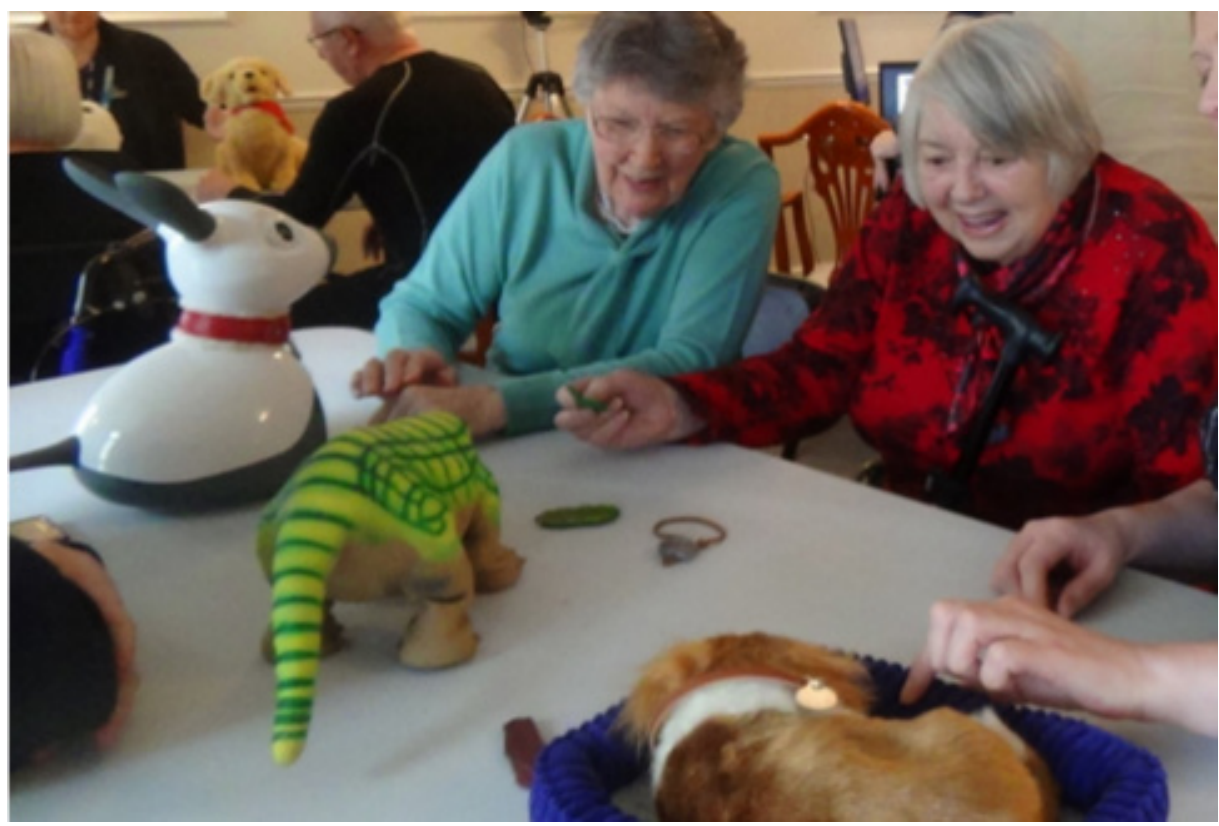

Figure 2 Interaction station 2. 
its head' (OP3 and OP1). Participants interacting with Paro sometimes displayed slight envy towards peers interacting with the Joy for All animals: 'you've done more with that cat than I got to do' (OP11).

Despite enjoying interactivity of available robots, older people also expressed a desire for command response from robots during free interactions. The commands each animal received varied. Those directed at the Joy for All dog were based on expectations of live dogs, with participants requesting 'high five' (OP3 and OP4), 'give paw' (OP3, OP5, OP8, OP10, OP15 and OP17) or 'lie down' (OP5), on 11 occasions. The Joy for All cat received similar requests, including 'can you wag your tail?' (OP3, OP1 and OP8). Miro mainly received directional commands, such as 'turn around!' (OP5 and OP6, OP10 and OP11, OP13, OP15 and OP17 and OP18) and 'stop, turn, turn left, turn left' (OP13), and Pleo received requests to play and eat: 'open wide, open wide, open up, that's it!' (OP13). The participants also repeatedly asked robots to 'look at me' (OP5, OP7, OP16 and OP15), suggesting that facial tracking and eye contact could be a future interactivity improvement; Paro and the Joy for All animals received praise as 'special' for 'looking right at' the he participant (OP2, OP4, OP13 and OP17). Most frustration was noted commanding the non-interactive Perfect Petzzz dog, with 15 participants requesting or commanding the dog to 'wake up' (OP1-OP6, OP9OP13 and OP16-OP18) or 'open your eyes' (OP5 and OP6, OP8 and OP9, OP12 and OP16). The participants reported limited appeal in an animal without responses, suggesting the non-interactive dog appeared 'dead' (OP17).

The participants also demonstrated desire for robot speech, comparing devices to the resident budgie and asking 'talk to me good boy' (OP7) because it would 'be better than talking to myself' (OP7). Another participant commented 'it's the company (sic) I talk to the furniture! (sic) if you live alone you often don't hear voices' (OP13) and 'I like to talk to things (sic) I think I just like to hear a voice' (OP14). Another spoke to Pleo, saying, 'I wish you could talk, yes I wish you could talk' (OP16). Similarly, on 11 occasions, participants confused Miro's electronic noises (not recognisable as specific animal vocalisations) with language, repeating, 'what are you saying?' (OP5), 'you're trying to talk aren't you?' (OP17) and 'I don't know if it's actual words or not' (OP14). On understanding Miro's noises were not 'actual words', one participant described the robot as 'a dead loss' (OP17).

Nonetheless, the participants still initiated conversation with non-speaking animals: 'what can we call you? We can call you Dino. It's not very original (sic), Dino, do you want to play again or eat?' (OP6). This sometimes resulted in disappointment when devices failed to respond verbally: 'you won't be much use to me if you don't talk to me' (OP9) and 'he doesn't talk back though,' 'can it hear? It's got no ears!' 'If he can't hear, he can't talk to me' (OP16).

\section{Familiarity}

This theme represents participants' desire for companion robots to be realistic and familiar in form, and emerged from codes: realistic animal, familiarity, comparison to real animals, reminiscence, life simulation and toys. Evidence arose on 71 occasions.

The participants commented on preferring cats or dogs, as what they had 'always had' (OP13 and OP17) and were 'used to' (OP8). The realistic, familiar options available also elicited comparisons to real animals, on 25 occasions with the Perfect Petzzz dog and the Joy for All cat and dog. The participants compared devices to previous pets, such as 'this one's like Harry' (OP5) or discussed the benefits of robot alternatives as being 'far easier' (OP3) because 'you don't have to take it out (sic) and clean up after it' (OP8) and 'it won't malt' (OP4). Familiar animals also prompted reminiscence on 12 occasions, probably due to greater relatability, such as 'I had (sic) Yorkshire terrier, tiny terrier, used to get lagged in the mud' (OP8). Only one occasion was negative: one participant had experienced 'a dead cat in the water off the pier when I was about 9' (OP5).

In contrast, unfamiliar forms were perceived by older people as 'a toy' (OP1) and more infantilising. During interactions with Miro and Pleo, one participant discussed preference for 'something, that to me, looks like something we've had, like dogs and cats and things, we've had dogs and cats you see' (OP10). The participants showed clear preference for familiar forms and realistic design over unfamiliar forms when both were available: 'that is realistic (dog), we're not very likely to come into contact with one of them (seal)' (OP5). The participants suggested seals were incongruent with their context, believing seals belong 'on the ice floats' (OP4) or 'eaten with pepper sauce' (OP4). The familiar animals were most often the devices praised for looking 'realistic' (OP3) or behaving in a way that appeared 'very real' (OP5).

Additionally, the breathing feature of the Perfect Petzzz dog was well received: 'it's fascinating to watch him breathing' (OP15). It appears any feature increasing the 'realness' of a companion was beneficial. Participants reported life-simulation features such as the breathing made the robots look 'living' (OP17). This feature was commented on 13 times and as often a source of conversation between participants.

\section{Shell design}

This theme arose on 89 occasions through codes: realistic animal, physical features, shell-type, favouritism, preference, texture and likeability. The evidence strongly suggested older people preferred soft, furry companion robots but also favoured big eyes. Participants did prefer features making animals appear more realistic, as discussed previously.

Paro's eyes were specifically commented on positively by six older people. The 'big eyes' (OP1 and OP4) were described as 'cute' (OP2) and appeared to draw participants towards the seal: 'ohhh look at your eyes!' (OP11). 
The participants also particularly appreciated Paro's prominent eyelashes: 'ladies will wish they had lashes like him!' (OP6). Other large eyes also received praise, including Furby's animated eyes, which were particularly 'captivating' (OP16).

Older people praised animals with fur for cuddliness and suggested, in response to non-furry options, that they 'want something (sic) you could smooth and it feels like an animal, you know, like that (Joy for All) cats got fur' (OP10). On 11 occasions, the participants responded negatively to plastic shells of Pleo and Miro, as they did not 'feel quite as friendly' (OP11). In contrast, Paro's fur was described as 'lovely' (OP8) and 'soft' (OP11). While participants appeared to acknowledge that Paro possessed softer fur than alternative furry animals, the Joy for All cat's fur was praised for being less pristine. The participants suggested the cat 'looks a bit bedraggled' (OP7), which resulted in time spent brushing and grooming. One participant suggested the fur looked 'so real' (OP1), suggesting the longer, shaggier coat felt more congruent with cat expectations.

\section{Ownership}

This theme arose on 30 occasions through codes naming, ownership and personalisation and represented older people demonstrating some attachment towards robots during free interactions.

Naming was thought to relate to ownership, as naming a live animal occurred with possession and signified a developing relationship. ${ }^{38}$ Older people sometimes used names of previous pets, such as 'Milo' (OP1) because 'they've got a cat called Milo' (OP3). Other participants chose generic names, such as 'Fido' (OP11) or 'Tigger' (OP4), while some got creative with names like 'Shandy' (OP7) because the dog 'is a mixture' (OP7). Once older people had allocated a name, it endured throughout their interaction: 'are you wagging your tail for me Shandy?' (OP7). Naming occurred mostly with the Joy for All cat and dog.

Further evidence of ownership came from a code of the same name. Ten older people commented on acquiring a robot during free interactions, such as 'do you know, I'd love this (cat), I'd love this in my apartment' (OP2). Another suggested 'the service should have one (Joy for All dog)' (OP6) with peers commenting in agreement, 'we'll all go out and buy one now!' (OP17). Of all occurrences, ownership was shown only towards the Joy for All cat and dog, suggesting good acceptability of these two devices.

We felt personalisation related to ownership, as wanting to adapt a robot for personal use implies a desire to keep it. Evidence of personalisation was not prolific during free interactions, with hints of personalisation being desired occurring only twice. One participant enjoyed the Joy for All dog but requested a larger size as 'I don't do little doggies' (OP16). The participant requested it 'look like a golden retriever' because 'it's the only dog we've ever known' (OP16). It is possible that evidence was limited during free interactions as participants were unaware of the possibility.

\section{Section 2: focus group results}

This section presents the focus groups results as a numerical comparison between end users and developers, to provide a clear understanding of any differences between the two groups. The features presented represent the most prevalent themes during content analysis of responses to the key questions (box 1). For both groups, an overall score was calculated for each feature (n participants responding positively minus $\mathrm{n}$ participants responding negatively). The difference between roboticists and older people's opinions for each feature was then calculated. Examples of focus group responses for comparison are also provided for greater depth of understanding.

Table 1 compares opinions of older people and roboticists towards the design of companion robots specifically for older people. The largest divergences in opinions were noted for scores for realistic aesthetic, robots talking human language, personalisation of robots and familiar form. Older people and roboticists seem to agree on the need for interactivity and soft fur in response to key questions 1 and 2 (box 1). There also appears to be some agreement between the two groups on inclusion of life-simulation features and mythical design, although older people were generally more positive towards life simulation and more negative towards mythical design. Some participants did not respond to every feature, resulting in lower numbers of responses for some features. Familiarity, life simulation and mythical design received lower responses, possibly suggesting these features were less important, and thus participants felt less inclined to comment. However, this could also derive from the semistructured nature of the focus groups, where realistic, familiar or mythical designs were all discussed in relation to key question 10.

The preferred animal among older people in response to key question 8 was the Joy for All cat, with 9/17 (53\%) participants selecting this animal (figure 3), followed by the Joy for All dog. Paro, Miro and the homemade hedgehog were not selected by any older person. The preferred animal among roboticists was Paro (11/18), followed by Pleo the dinosaur, then the homemade hedgehog. The Joy for All dog and cat, Miro, the Perfect Petzzz dog and Furby were not selected by any roboticists, and some roboticists did not select any of the available animals.

Table 2 provides examples of the different views of older adults and roboticists during focus group discussions; further examples can be found in online supplementary file 1 .

\section{Section 3: relationship between free interaction and focus group data}

This section explores how the themes arising during unprompted, free interaction support the validity of the prompted focus group results (figure 4): all older people 


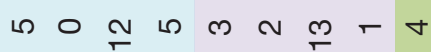

- NE

0
0

N

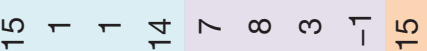

$\stackrel{T}{N} \circ \wedge \sim \underset{T}{T}$

$\stackrel{+}{N}=\infty-a \wedge$

LO O N

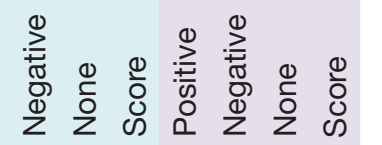

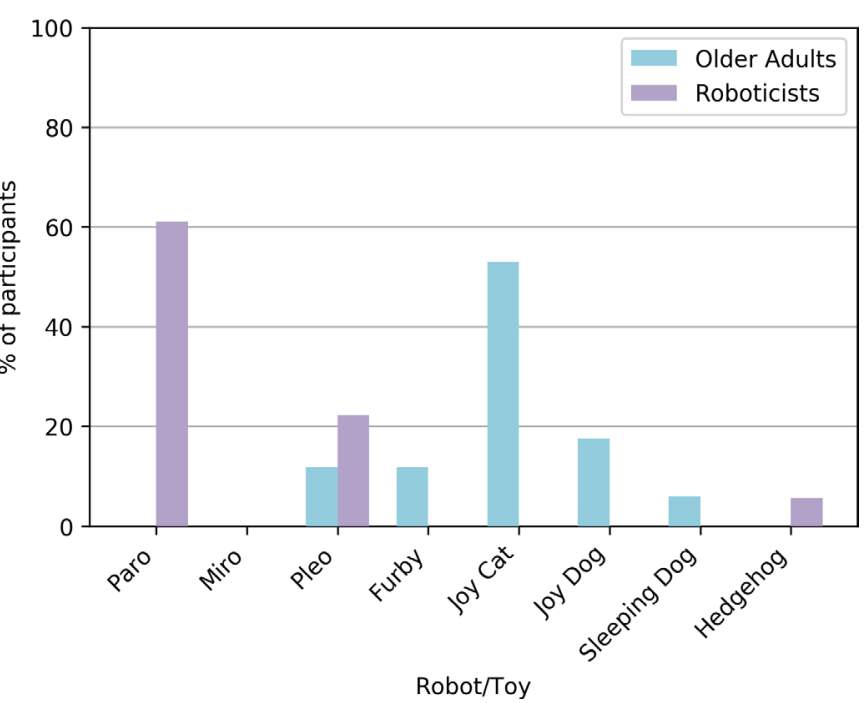

Figure 3 Choice of robot/toy for use with older people, shown by participant group.

who discussed interactivity $(15 / 17,88.24 \%)$ desired this feature for a robot pet. As seen in section 1, this feature was highly valued by older people during free interactions, with many participants desiring additional interaction, such as obeying commands and talking. In the the focus group theme of talking, 12/17 (71\%) older people felt positively towards robot speech.

The free interaction theme familiarity supports the focus group results where all older people who commented $(4 / 17,24 \%)$ preferred familiar forms and $12 / 17(71 \%)$ and realistic or life-like appearance, with only 1/17 (6\%) older people responding negatively to life-like appearance (thus, $92.31 \%$ of responses were positive). The higher percentage of non-responses to familiarity could suggest participants felt less strongly about this feature and thus less inclined to comment. However, the qualitative results from free interactions would dispute this, with very strong support arising in favour of a familiar animal. Therefore, it may instead be possible that participants did not necessarily distinguish between realistic and familiar (as realistic, unrealistic and mythical were the words used within the key questions).

The free interaction theme on shell type and clear preference for soft fur are congruent with focus group results where $12 / 17$ (71\%) older people preferred soft fur, while only $1 / 17(6 \%)$ disagreed (92\% of responses positive). Life simulation was not discussed at length during free interactions, although the Perfect Petzzz breathing feature was well received. This feature also had lower response rates during focus groups. The lower response rate for this feature could again suggest that, while life simulation may be desirable, supported through decisive responses ( $100 \%$ of responses were positive), it may be less of a priority, with $12 / 17(71 \%)$ older people not providing opinions. Despite the limited direct discussion during free interactions, the potential inclusion of this feature is supported by the familiarity theme, whereby any aesthetic or technological features increasing the realness 
Table 2 Examples of evidence from each group during focus group discussions

\begin{tabular}{|c|c|}
\hline & Example evidence \\
\hline Theme & Older people \\
\hline Interactivity & $\begin{array}{l}\text { 'If you're sat there on your own, you want some } \\
\text { reaction'. (OP6) } \\
\text { 'That one (Joy for All cat) is almost perfect, but } \\
\text { perhaps if you could say, do you want to play, } \\
\text { and then it could then do something, a little bit } \\
\text { more interactive'. (OP13) }\end{array}$ \\
\hline
\end{tabular}

Soft fur

'Day to day cleaning, you could wipe over it (Pleo), furry thing would be harder'. (OP5) 'Fur I think so. The plastic I found very cold, not something you would, sorta, cuddle'. (OP13)

Talking '(animals) don't talk, there are sounds that
creatures make'. (OP6)
'For older people living on their own in
particular, we all talk to ourselves anyway, you
don't feel so stupid if you talk to something
that responds to you'. (OP13)

Personalisation 'If it was knitted, it wouldn't be able to move its eyes and mouth'. (OP5)

'It's quite a good idea, yeah I do, someone who's got a particular animal' 'We were talking about colours, I like that one, she's always had black cats, It would be nice to have a choice of different colours'. (OP13)

$\begin{array}{ll}\text { Realistic } & \text { 'For someone who's always had animals, they } \\ & \text { feel that loss, so for them, something realistic } \\ \text { that they could interact with'. (OP1) } \\ \text { 'as long as it's got big eyes and attractive I } \\ \text { don't mind'. (OP17) }\end{array}$

Familiarity 'because they (cat and dog) are more domesticated animals, whereas a seal you wouldn't have a seal in your home'. (OP1) 'I think if you'd had a cat or a dog, it would be better to have something you could relate to'. (OP12)

\begin{tabular}{|c|c|c|}
\hline Mythical & $\begin{array}{l}\text { 'That's a generation thing, kids would love it } \\
\text { but not here'. (OP1) } \\
\text { 'Maybe in } 5 \text { years time...'. (OP16) }\end{array}$ & $\begin{array}{l}\text { 'I also think something super unrealistic like the Furby } \\
\text { would be creepy as well, it's so bizarre you could } \\
\text { be turned off by it, it's weird, a baby seal, you're not } \\
\text { accustomed to the animal so whatever it does is just } \\
\text { cute'. (R8) } \\
\text { 'The mythical Furby looks right because you've got no } \\
\text { expectations, so you cannot do it wrong, you cannot } \\
\text { break expectations'. (R13) }\end{array}$ \\
\hline Life simulation & $\begin{array}{l}\text { 'Warmth under belly to keep your knees } \\
\text { warms!' (OP1) } \\
\text { 'If it was breathing, it would be almost a real } \\
\text { cat, and again, it's a soothing thing'. (OP14) }\end{array}$ & $\begin{array}{l}\text { 'I can feel on the dinosaur, coming from an engineering } \\
\text { point of view, with all that inside and trouble circulating } \\
\text { the air, you can feel it gets warm, but I think that's } \\
\text { actually a good thing, that you can feel, it's even more, } \\
\text { like lizard like, even more appearing like something'. (R6) } \\
\text { 'The problem is I think it has to be done well, and it's } \\
\text { really difficult to do well, it could end up creepy and } \\
\text { weird'. (R14) }\end{array}$ \\
\hline
\end{tabular}

\section{Roboticists}

'I think something passive, that doesn't make a lot of sounds, it could be stressful, too much (sic) You could have a sack that's warm and purrs'. (R3)

'I think it should have high level interaction, because it would keep the interaction longer as well, if you just have a pet like this with one or two features, it's done, it's limited'. (R9)

'I don't think so, because it isn't cleanable, if you wanted something to cuddle you could just buy a stuffed toy'. (R14)

'Nice and furry, you could kinda cuddle it'. (R18)

'from a technological point of view, speech should be left out of the equation, especially with elderly people, and people with dementia, they wouldn't have expressions or fully structured sentences which would get frustrating if the robot didn't understand'. (R1) 'I can see the appeal, (sic) a rudimentary conversation might be quite nice, as long as you didn't feel like a twit doing it'. (R11)

'That might ruin the illusion l'd say' 'if you've eaten like a chicken, if you've seen the actual process, you would not feel so good about it (sic), when you see the finished product without knowing how, it's sometimes better'. (R2)

'It would be amazing, it would give it a personal touch, it's like having a new (smartphone) and getting a new cover, people love that'. (R10)

'It would make more sense'. (R1)

'No (sic) if it's not realistic, you wouldn't be hoping it would be a real dog so'. (R16)

'for the elderly it should be something familiar'. (R2) 'I think because of uncanny valley it doesn't have to be something that we are used too'. (R7) 


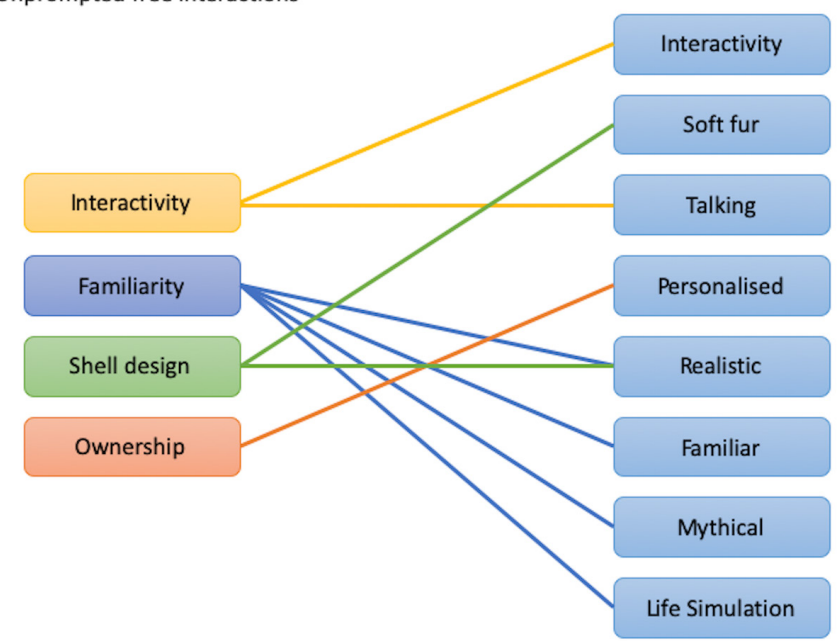

Figure 4 Mapping the relationship between older people's unprompted opinions and focus group themes.

of a pet appeared well received during unprompted free interaction.

While personalisation was not highly prevalent during free interaction, some evidence was seen within the ownership theme, with a participant requesting a golden retriever design. Within focus groups, 15/17 (88\%) older people felt positively towards personalisation, and only $1 / 17(6 \%)$ provided opposition $(94 \%$ of responses were positive). It is possible that personalisation garnered limited discussion during free interactions as participants were unaware it was possible. The range of suggestions of preferred animals on proposal of personalisation, however, would certainly suggest some benefit to this approach.

\section{DISCUSSION}

User-centred design is often cited as beneficial ${ }^{426}$ but rarely used in companion robot development. The differing preferences of end users and potential developers in our direct comparison demonstrated the importance of user-centred design when developing companion robots for older people. Our results justify additional effort for the reportedly difficult process of integrating user requirements into design ${ }^{29}$ and may aid acceptability of user-centred design in practice..$^{26}$ Some of our roboticists felt user involvement in development could damage illusions of the robot, perhaps helping explain the minimal use of this process. However, rather than damaging illusions, adopting a user-centred design may actually ensure that devices receive adequate acceptability to promote use. ${ }^{25}$ Future development of robots using user-centred approaches may result in more consistent positive outcomes than those previously reported for Paro. ${ }^{17} 1820{ }^{21}$ Implications of improved design, acceptability and use would be significant, given the potential benefits of companion robots for older people, those with dementia, and their family and care team. ${ }^{11-16}$ Our results suggest strong acceptability and preference of the Joy for All cat and dog and limited acceptability of Paro when these more familiar/realistic comparisons are available. This result is important, given a lack of comparison studies of companion robots ${ }^{39}$ and apparent selection bias towards Paro in research. ${ }^{10}$

Further to highlighting the value of user-centred design, this study provided initial insights on end-user design requirements. Older people and roboticists both saw interactivity as important. Older people wanted interactivity for companionship, fun and reduced loneliness through responsiveness. Some roboticists, on the other hand, raised concerns on overstimulating older people. Our older adults displayed little interest towards non-interactive animals whose lack of responsiveness appeared frustrating. This disinterest in unresponsive/inactive companions is congruent with the finding that an 'active' Paro was more engaging than an 'inactive' Paro. ${ }^{18}$ While interactivity appears essential, our results demonstrated that the advanced responsivity of Paro may be unnecessary. Despite having fewer technological abilities, the Joy for All cat was perceived as most interactive, most likely because of the greater range of movements available, including animated head and legs, rolling-over, blinking and cleaning movements. Therefore, the range and variety of responses may be more important than the sophistication of sensors a robot possesses.

Our older people were interested in companion robots understanding and responding to simple commands. Use of commands is only briefly mentioned in previous literature, ${ }^{32}$ and our findings appear contrary to a study ${ }^{40}$ that found no evidence for the importance of enjoyment or playfulness factors among community-dwelling older adults. Our group actively sought playfulness from robots, believing this would sustain enjoyment for longer. Responsiveness to simple commands such as 'paw' could be a consideration for future robot design. Interestingly, there were fewer command expectations for the Joy for All cat than other robots, perhaps due to a reduced association between live cats and training versus live dogs. These expectations could be used to support use of an unfamiliar form such as Paro, whose design was aimed at reducing expectations. ${ }^{41}$ However, older people still displayed command expectations for Pleo, Miro and Paro (unfamiliar forms), disputing this theory. One could speculate that the cat's larger quantity of movements results in a reduced need to command actions.

Older people also positively evaluated the potential for human speech from a companion robot. These results contradict the suggestion that, congruent with the uncanny valley theory, human acceptability of sounds depends on the realism of the context. ${ }^{42}$ In one study, ${ }^{43}$ participants related less to an AIBO dog beeping than a computer emitting an identical sound, perhaps due to contradiction in context between a dog and a beeping noise, thus suggesting that animal sounds would be most acceptable for animal robots. Our results, however, indicated positive attitudes towards speech capabilities for 
provision of company. Frennert and Östlund ${ }^{33}$ reported that developers were influenced by stereotypical perceptions of older people as lonely and fragile but failed to incorporate requirements of participating older people into design. Our group of older people thought loneliness could be eased through devices capable of simple conversation. This could be a user-driven improvement to currently available companion animals if our results are replicated in wider samples. It is possible, however, that this feature will be evaluated differently in possible future research with a sample of cognitively impaired older people. Our participants were cognitively intact and therefore aware of the artificial nature of the robots or toys; older people with dementia may find the incongruence of human speech from an animal less acceptable.

Eye contact was a further improvement desired by older people, some of whom were disappointed when robots failed to look towards them. Gaze following may increase social relevance of the robot. This may be particularly true when eye movement is intentional rather than random. ${ }^{44}$ While the preprogrammed movements of the Joy for All cat were positively evaluated, intentional gaze following may be an improvement for optimal social companionship. The importance of improving sociability for robot acceptance was noted before ${ }^{45}$ and this addition of apparent social behaviour could improve acceptability.

Most older people preferred soft, cuddly fur for the outer shell. Our group of roboticists generally agreed, although both groups raised concerns regarding hygiene in comparison to a hard shell. This corroborates previous findings on care providers' preferences for robots aimed at their older service users, ${ }^{34}{ }^{46}$ although others have reported older people's preference for mechanical design on robots. ${ }^{28}$ These results may reflect the broader range of socially assistive robots used (machine-like, mechanical, human-like and animal-like robots); however, results generally imply a robot should indeed be recognisable as robotic. ${ }^{28}$ One study ${ }^{21}$ also reported a family member demonstrating stigma towards his father interacting with soft toys, suggested potential gender barriers with soft, cuddly robots. Our study found no notable difference between men and women and suggests that companion robots for this market should use soft fur in the design. Providing the optimum tactile characteristics are particularly important considering evidence suggests that touch is one of the most important modalities of interaction for dementia patients, creating a natural method to engage with animaloid robots. ${ }^{47}$

Considering the importance of tactile characteristics, ${ }^{46}$ a further feature for consideration in future development is life simulation, another capability positively evaluated by older people but lacking from current examples, including Paro. Our research supports the previously reported ${ }^{46}$ assumption of care providers that a simulated heartbeat would be a valuable addition to Paro, but additionally demonstrates that older people themselves also valued life-simulation features, including simulated heartbeat, simulated breathing and the feeling of purring.
Older people even suggested warmth as an additional feature. This result appears congruent with older adults' desire for a realistic, life-like companion.

A realistic, familiar animal form was a definite aesthetic requirement for our group of older people. This was also reflected in their choice of Joy for All cat as their preferred device, as a familiar, realistic option, with Paro not selected by any older adult. Previous research focusing on opinions of care providers revealed criticism of Pleo for lack of familiarity, ${ }^{34}$ while the intentionally unfamiliar Paro ${ }^{41}$ is the most often used companion robot in research. ${ }^{10}$ The end users in our research thought that Paro, like Pleo, was too unfamiliar. The most familiar animals, the Joy for All cat and dog, were preferred for being more relatable and congruent with the contexts in which older people lived. The unfamiliar forms appeared incongruent and infantilising, perhaps explaining the tension Lazar $e t a l^{32}$ found towards their selection of unfamiliar animals.

This is relevant insofar as some companion robots, such as Paro, are intentionally designed using unfamiliar forms to prevent the robots from failing to meet expectations. ${ }^{41}$ Most of our roboticists followed this line of thinking and responded negatively to familiar animals, unsurprisingly selecting Paro as their preferred companion robot. It is further likely the roboticists appreciated the advanced technical capabilities of Paro, but our study suggests such sophistication may be unnecessary for older people. Research conducted 19 years ago also suggested older people disliked the feel and behaviour of a robot cat compared with real cats $^{47}$; however, currently available robotic cats are likely more realistic than the Tama OMRON Corp cat used in that study.

The preference for realistic and familiar robots may result from relatability, with older people perhaps having personal experience of cats and dogs, given the prevalence of ownership of these species. ${ }^{48}$ Familiar animals may provide recognisable potential for a loving relationship. Even individuals without personal pet ownership experience will have likely witnessed others with pets, and therefore the familiar form of a dog or cat is symbolic of that potential bond and relationship. The tendency for our group of older people to name the Joy for All cat and dog more often than alternatives suggests familiarity may additionally help facilitate a sense of ownership. Thus, our results imply that, rather than being problematic, ${ }^{41}$ memories and schemas of familiar animals may actually be beneficial. A further implication of familiar companion robots relates to reminiscence theory, which suggests benefits of reminiscence for older people, including decreased depression. ${ }^{49}$ Reminiscence therapy uses memories, feelings and thoughts from the past to facilitate pleasure.$^{50}$ Evidence of reminiscence was found in our study and seems congruent with this theory, as memories of past pets and animals were shared with positive affect. It is therefore possible that familiar companion robots would have additional well-being benefits, particularly for individuals with dementia. 
The possibility of personalisation was also positively perceived by older people and thus could be a consideration for future robot design. Personalisation has been mentioned in previous research ${ }^{28} 34$ but has not been explored directly with end users. Our older people positively evaluated a more person-centred approach to robot aesthetics, praising the potential to interchange robot 'skins' to match personal preference. It is possible personalised robots would be more acceptable than a single design for all users. This could alleviate some disparity in response to Paro, as seen in previous RCT research. ${ }^{22}$

In contrast, our roboticists underestimated the value of personalisable aesthetics and failed to predict older people's desire for human speech and life-simulation features. The transcript evidence suggests roboticists had an awareness of Mori's uncanny valley hypothesis. ${ }^{51}$ This is not surprising, given their field of interest, and it is possible that this, as well as related literature, had influenced roboticists' views on robot design to favour unrealistic and unfamiliar forms and to undervalue life simulation features that would undoubtedly increase the realistic impression of a robot.

Although our study was limited by recruiting older people from just one setting and roboticists from one university (although from varied educational and occupational backgrounds), we found marked differences in their views that need to be accounted for in the development of companion robots. If creative methods of coproduction are used, ${ }^{52}$ both groups would need to think more about why they liked certain features, and it is likely they would develop a new product that would be owned by this codesign group. Although there are no guarantees, a product so designed might have a higher chance of being liked by the wider population of older people.

Our study recruited older people from a retirement complex, and the generalisability of their views to care home residents is limited. Our finding of the acceptability of such devices among a more independent sample is in contrast to previous research, which implied more independent older people felt 'too able' to use robots. ${ }^{28}$ Thus, there may be a market among this more independent sample that has previously been underestimated.

Another limitation of our study was the short interaction time of 10 minutes at each station, providing initial preferences. Research has suggested acceptance should be measured over longer periods of use, allowing for familiarisation and more informed attitudes towards the device, which may be more predictive of actual use. ${ }^{53}$ Future longitudinal research is therefore required, exploring how these initial preferences develop over time, to assess any differences in loss of engagement or well-being outcomes. Our interaction period was, however, longer than previous research where participants only interacted with each robot for $1 \mathrm{~min} .{ }^{34}$

Our study's smaller group sizes compared with previous research ${ }^{34}$ may have limited the influence of social desirability bias or group dynamics. The small sample size, as well as the small number of responses to some features during focus groups, is a further limitation. On the other hand, use of qualitative, free interaction transcriptions increases confidence in our focus group results, even where response numbers were low, as preferences were often evident through unprompted interaction.

An important strength of the current study is the active participation of older people themselves. Some previous research exploring design features of companion robots for older people focused mainly on care provider opinions. ${ }^{28} 46$ Our research has provided support for some previously identified features but furthered this evidence base through identification of design features previously unthought of by care providers. A further strength includes the use of a range of robots and toys, some specifically designed for older people, unlike previous related literature, ${ }^{32}$ providing a varied array of features of interest and allowing older people to provide truly informed opinions.

\section{CONCLUSION}

We have provided empirical support for the necessity and value of incorporating user-centred design in the development of companion robots targeted at older people. While user-centred design has been recommended previously, there has been little direct evidence to support this requirement. Our results demonstrate stark differences in preferences and requirement between older people and roboticists, suggesting that engaging the end user in the design and development of companion robots is essential. This study also began the process of researching companion robot design with end users themselves. The older people in our sample have suggested soft fur, interactivity and big, cute eyes as being priority features on a robot. Older people also strongly suggested the robot should take the form of a realistic, familiar animal, raising questions surrounding the design of the most well-researched companion robot, Paro. Further desirable functions were also identified that are not currently included as standard on companion robots, such as eye-contact, life-simulation features, personalisation, obeying commands and the potential for interactive language.

\section{Author affiliations}

${ }^{1}$ Faculty of Health and Human Sciences, University of Plymouth, Plymouth, UK ${ }^{2}$ Department of Nursing, Auckland University of Technology, Auckland, New Zealand ${ }^{3}$ Donders Centre for Cognition, Radboud University, Nijmegen, The Netherlands

Acknowledgements The authors thank Mrs M Jones for knitting and kindly supplying the hedgehog used in this research, Ms D Hubbard for assistance with participant recruitment, Kirsty Langstaff for assistance with data collection, Jake Gibson Shaw-Sutton and Khaian Marsh for assistance with data collection and recording equipment, and the EHealth Productivity and Innovation in Cornwall and the Isle of Scilly Project, which is in part funded by the European Regional Development Fund, for the loan of some of the robots used in this research.

Contributors All authors read and approved the manuscript. HLB designed the study; performed data collection; transcribed, analysed and interpreted the results; 
and led the production the manuscript. KJE transcribed the data, analysed and interpreted the results, and aided in the production of the original manuscript. RW supervised the project, provided expertise and advice towards the study conception and design, discussed the results and substantively revised the manuscript. ST supervised the project, provided expertise and advice towards the study conception and design, discussed the results and substantively revised the manuscript. RBJ oversaw participant recruitment and data collection, supervised the project, provided expertise and advice towards the study conception and design, discussed the results and substantively revised the manuscript.

Funding H. Bradwell's PhD was funded by a PhD studentship from the School of Nursing and Midwifery at the University of Plymouth. The robots used in this study were loaned from the School of Nursing and Midwifery and the Ehealth Productivity and Innovation in Cornwall and the Isle of Scilly (EPIC) project, which is part funded by the European Regional Development Fund (ERDF). All of the above were 'general funds' to fund study in this area of endeavour. The EPIC project funded publication costs as part of its dissemination and knowledge transfer to the EPIC community.

Competing interests None declared.

Patient consent for publication Not required.

Ethics approval Ethical approval was obtained from the Faculty of Science and Engineering ethics committee at the University of Plymouth. All participants provided full, written informed consent prior to the study.

Provenance and peer review Not commissioned; externally peer reviewed.

Data availability statement Data are available in a public, open access repository.

Open access This is an open access article distributed in accordance with the Creative Commons Attribution Non Commercial (CC BY-NC 4.0) license, which permits others to distribute, remix, adapt, build upon this work non-commercially, and license their derivative works on different terms, provided the original work is properly cited, appropriate credit is given, any changes made indicated, and the use is non-commercial. See: http://creativecommons.org/licenses/by-nc/4.0/.

\section{REFERENCES}

1. Abdi J, Al-Hindawi A, $\mathrm{Ng} \mathrm{T}$, et al. Scoping review on the use of socially assistive robot technology in elderly care. BMJ Open 2018;8:e018815

2. Garcon L, Khasnabis C, Walker L, et al. Medical and assistive health technology: meeting the needs of aging populations. Gerontologist 2016;56(Suppl 2):S293-302.

3. Broadbent E, Stafford R, MacDonald B. Acceptance of healthcare robots for the older population: review and future directions. Int $J$ Soc Robot 2009;1:319-30.

4. Moyle W, Jones C, Pu L, et al. Applying user-centred research design and evidence to develop and guide the use of technologies, including robots, in aged care. Contemp Nurse 2018;54:1-3.

5. Steptoe A, Deaton A, Stone AA. Subjective wellbeing, health, and ageing. Lancet 2015;385:640-8.

6. Farrand P, Matthews J, Dickens C, et al. Psychological interventions to improve psychological well-being in people with dementia or mild cognitive impairment: systematic review and meta-analysis protocol. BMJ Open 2016;6:e009713.

7. Broekens J, Heerink M, Rosendal H. Assistive social robots in elderly care: a review. Gerontechnology 2009;8:94-103.

8. Moyle W, Cooke M, Beattie E, et al. Exploring the effect of companion robots on emotional expression in older adults with dementia: a pilot randomized controlled trial. J Gerontol Nurs 2013;39:46-53.

9. Valentí Soler M, Agüera-Ortiz L, Olazarán Rodríguez J, et al. Social robots in advanced dementia. Front Aging Neurosci 2015;7:133.

10. Pu L, Moyle W, Jones C, et al. The effectiveness of social robots for older adults: a systematic review and meta-analysis of randomized controlled studies. Gerontologist 2019;59:e37-51.

11. Jøranson N, Pedersen I, Rokstad AMM, et al. Effects on symptoms of agitation and depression in persons with dementia participating in robot-assisted activity: a cluster-randomized controlled trial. J Am Med Dir Assoc 2015;16:867-73.

12. Wada K, Shibata T, Saito T. Psychological and social effects of one year robot assisted activity on elderly people at a health service facility for the aged. Proceedings of the 2005 IEEE International Conference on Robotics and Automation, Barcelona, Spain: IEEE, 2005.

13. Saito T, Shibata T, Wada K. Relationship between interaction with the mental commit robot and change of stress reaction of the elderly.
Proceedings 2003 IEEE International Symposium on Computational Intelligence in Robotics and Automation, Kobe, Japan, 2003:119-24.

14. Liang A, Piroth I, Robinson $\mathrm{H}$, et al. A pilot randomized trial of a companion robot for people with dementia living in the community. $J$ Am Med Dir Assoc 2017;18:871-8.

15. Petersen S, Houston S, Qin $\mathrm{H}$, et al. The utilization of robotic pets in dementia care. J Alzheimers Dis 2017;55:569-74.

16. Robinson $\mathrm{H}$, MacDonald $\mathrm{B}$, Broadbent $\mathrm{E}$. Physiological effects of a companion robot on blood pressure of older people in residential care facility: a pilot study. Australas J Ageing 2015;34:27-32.

17. Misselhorn C, Pompe U, Stapleton M. Ethical considerations regarding the use of social robots in the fourth age. GeroPsych 2013;26:121-33.

18. Moyle W, Jones CJ, Murfield JE, et al. Use of a robotic seal as a therapeutic tool to improve dementia symptoms: a clusterrandomized controlled trial. J Am Med Dir Assoc 2017;18:766-73.

19. Robinson $\mathrm{H}$, MacDonald $\mathrm{B}$, Kerse $\mathrm{N}$, et al. The psychosocial effects of a companion robot: a randomized controlled trial. J Am Med Dir Assoc 2013;14:661-7.

20. Thodberg K, Sørensen LU, Christensen JW, et al. Therapeutic effects of dog visits in nursing homes for the elderly. Psychogeriatrics 2016;16:289-97.

21. Robinson $\mathrm{H}$, MacDonald BA, Kerse N, et al. Suitability of healthcare robots for a dementia unit and suggested improvements. J Am Med Dir Assoc 2013;14:34-40.

22. Moyle W, Jones $\mathrm{C}$, Murfield J, et al. Using a therapeutic companion robot for dementia symptoms in long-term care: reflections from a cluster-RCT. Aging Ment Health 2019;23:329-36.

23. Odetti L, Anerdi G, Barbieri MP. Preliminary experiments on the acceptability of animaloid companion robots by older people with early dementia. Proceedings of the 29th Annual International Conference of the IEEE EMBS, Lyon, France, 2007:1816-9.

24. Tamura T, Yonemitsu S, Itoh A, et al. Is an entertainment robot useful in the care of elderly people with severe dementia? J Gerontol A Biol Sci Med Sci 2004;59:M83-5.

25. Heerink M, Kröse B, Evers V, et al. Assessing Acceptance of Assistive Social Agent Technology by Older Adults: the Almere Model. Int J Soc Robot 2010;2:361-75.

26. Chammas A, Quaresma M, Mont'Alvão C. A closer look on the user centred design. Procedia Manuf 2015;3:5397-404.

27. Orrell M, Hancock GA, Liyanage KCG, et al. The needs of people with dementia in care homes: the perspectives of users, staff and family caregivers. Int Psychogeriatr 2008;20:941-51.

28. Pino M, Boulay $M$, Jouen $F$, et al. 'Are we ready for robots that care for us?' Attitudes and opinions of older adults toward socially assistive robots. Front Aging Neurosci 2015;7:141.

29. Green A, Hüttenrauch H, Norman M. User centered design for intelligent service robots. Proceedings 9th IEEE International Workshop on Robot and Human Interactive Communication, Osaka, Japan, 2000:161-6.

30. Sandoval E, Penaloza C. Children's knowledge and expectations about robots: a survey for future user-centered design of social robots. 7th ACM/IEEE International Conference on Human-Robot Interaction, Boston, MA, 2012:107-8.

31. Wu Y-H, Cristancho-Lacroix V, Fassert C, et al. The attitudes and perceptions of older adults with mild cognitive impairment toward an assistive robot. J Appl Gerontol 2016;35:3-17.

32. Lazar A, Thompson H, Piper AM. Rethinking the design of robotic pets for older adults. Proceedings of the 2016 ACM Conference on Designing Interactive Systems, Brisbane, QLD, Australia, 2016.

33. Frennert S, Östlund B. Review: seven matters of concern of social robots and older people. Int J Soc Robot 2014;6:299-310.

34. Heerink M, Albo-Canals J, Valenti-Soler M. Exploring requirements and alternative PET robots for robot assisted therapy with older adults with dementia. Proceedings of the 5th International Conference on Social Robotics, Bristol, UK, 2018:104-15.

35. Vaismoradi M, Turunen $\mathrm{H}$, Bondas T. Content analysis and thematic analysis: implications for conducting a qualitative descriptive study. Nurs Health Sci 2013;15:398-405.

36. Mayring P. Qualitative content analysis. Forum: Qual Soc Res 2000;1.

37. Elo S, Kyngäs $\mathrm{H}$. The qualitative content analysis process. J AdV Nurs 2008;62:107-15.

38. Bayne K. Development of the human-research animal bond and its impact on animal well-being. Ilar J 2002;43:4-9.

39. Kachouie R, Sedighadeli S, Khosla R, et al. Socially assistive robots in elderly care: a Mixed-Method systematic literature review. Int J Hum Comput Interact 2014;30:369-93.

40. Klamer T, Allouch SB. Acceptance and use of a social robot by elderly users in a domestic environment. 2010 4th International Conference on Pervasive Computing Technologies for Healthcare, Munich, Germany, 2010:22-5. 
41. Shibata T, Wada K. Robot therapy: a new approach for mental healthcare of the elderly - a mini-review. Gerontology 2011;57:378-86.

42. Jones T, Lawson S, Mills D. Interaction with a zoomorphic robot that exhibits canid mechanisms of behaviour. 2008 IEEE International Conference on Robotics and Automation, 2008:2128-33.

43. Komatsu T, Yamada S. How does the agents' appearance affect users' interpretation of the agents' attitudes: experimental investigation on expressing the same artificial sounds from agents with different appearances. Int J Hum Comput Interact 2011;27:260-79.

44. Abubshait A, Wiese E, Human YL. You look human, but act like a machine: agent appearance and behavior modulate different aspects of Human-Robot interaction. Front Psychol 2017;8:1393.

45. de Graaf MMA, Ben Allouch S, van Dijk JAGM. Why would I use this in my home? a model of domestic social robot acceptance. HumanComputer Interaction 2019;34:115-73.

46. Jung MM, van der Leij L, Kelders SM. An exploration of the benefits of an Animallike robot companion with more advanced touch interaction capabilities for dementia care. Front ICT $2017 ; 4: 1-11$
47. Shibata T, Tanie K. Influence of a priori knowledge in subjective interpretation and evaluation by short-term interaction with mental commit robot. Proceedings 2000 IEEE/RSJ International Conference on Intelligent Robots and Systems, Takamatsu, Japan, 2000:169-74.

48. Murray JK, Browne WJ, Roberts MA, et al. Number and ownership profiles of cats and dogs in the UK. Vet Rec 2010;166:163-8.

49. Hsieh H-F, Wang J-J. Effect of reminiscence therapy on depression in older adults: a systematic review. Int J Nurs Stud 2003;40:335-45.

50. Syed Elias SM, Neville C, Scott T. The effectiveness of group reminiscence therapy for loneliness, anxiety and depression in older adults in long-term care: a systematic review. Geriatr Nurs 2015;36:372-80.

51. Mori M, MacDorman K, Kageki N. The Uncanny Valley [From the Field]. IEEE Robot Autom Mag 2012;19:98-100.

52. Easton K, Potter S, Bec R, et al. A virtual agent to support individuals living with physical and mental comorbidities: Co-Design and acceptability testing. J Med Internet Res 2019;21:e12996.

53. Wu Y-H, Wrobel J, Cornuet M, et al. Acceptance of an assistive robot in older adults: a mixed-method study of human-robot interaction over a 1-month period in the living lab setting. Clin Interv Aging 2014;9:801-11. 